\title{
EDITORIAL
}

\section{The perils of plastic}

A 'round-robin' spam e-mail that is circulating on servers worldwide claims that drinking bottled water that has been left in a warm car can cause breast cancer. Is this warning just an urban myth or does it hold a grain of truth? The FDA, it seems, is erring on the side of caution; earlier this year, the organization revised its position on the safety of bisphenol A (BPA), a chemical used in the manufacture of plastics. Previously deemed safe for food-contact use, the FDA has now expressed "some concern" about the potential health risks that BPA poses to fetuses, infants and young children.

What exactly is BPA and why has its use caused alarm? First synthesized in 1905, BPA has since become a key component in the production of plastics, including polycarbonate and epoxy resins. Polycarbonate is a clear, heat-resistant, shatter-proof material, properties that make it ideal for the manufacture of drinks bottles, particularly those used by young children and infants. Epoxy resins are also used by the food and beverage industry-they provide the protective coating found inside many metal-based cans. Standard toxicology tests supported the safety of BPA and the FDA approved it for food-contact use in the 1960s. Over the past few years, however, concern has mounted that low-level environmental exposure to BPA might disrupt normal functioning of the endocrine system.

The term 'endocrine disruption' was coined in the early 1990s. Endocrine disruptors comprise a diverse group of industrial chemicals that exert numerous developmental and functional effects on the endocrine system via multiple biological pathways. Many of these chemicals mimic the effects of endogenous hormones, such as estrogen. BPA and other endocrine-disrupting chemicals have been implicated in obesity, neurological deficits, reproductive dysfunction and cancer. In addition, perfluorooctanoic acid (PFOA) and perfluorooctane sulfonate (PFOS) - common household chemicals found in 'nonstick' and waterproof materials-have recently been linked to thyroid disease.

The Endocrine Society has recognized the potential problems associated with the widespread use of industrial chemicals. In June 2009, the society published the findings of a task force commissioned to investigate the mechanisms of action and potential health risks of endocrine disruptors (Diamanti-Kandarakis, E. et al.
Endocr. Rev. 30, 293-342; 2009). The task force highlighted a number of unique features of endocrinedisrupting chemicals that should be considered before robust recommendations can be put in place. The adverse effects of exposure to endocrine disruptors extend beyond the individual: the fetus and newborn infants are particularly vulnerable and multigenerational effects have been described. The biological consequences of endocrine disruptors can manifest at low doses and many years after the initial exposure; furthermore, individuals are simultaneously exposed to multiple compounds on a daily basis. Traditional toxicology testing does not take these factors into account.

The initial focus is on minimizing the risk to children and infants. The FDA is actively supporting industry efforts to find alternatives to BPA and to reduce the levels of BPA in the linings of cans and other packaging. In addition, many retailers are phasing out the sale of BPA-containing products aimed at the childcare market and BPA-free bottles and beakers are already available. Although such efforts are laudable, BPA is just the tip of the iceberg. The plastics industry has a responsibility to ensure that its products are safe; companies should, therefore, be mandated to fund independent epidemiological and mechanistic research into the potential risks that industrial chemicals pose to the endocrine system. Furthermore, the FDA and its international counterparts should prioritize such research; at a global level, even a small risk could have a considerable impact on the health of millions of people.

Is society compromising its health for the conveniences of modern living? Industrial chemicals, such as BPA, are literally everywhere: in homes, in the workplace, even the great outdoors. They cannot possibly all be avoided. Given the current recommendations of the FDA and the Endocrine Society, a multidisciplinary approach is clearly needed-one that involves scientists, clinicians, policy makers and the chemicals industry-with the aim of gathering reliable data to form the basis of national and international public-health policies. In the meantime, the use of plastics and other man-made substances should be closely monitored in groups known to be at the greatest risk. Perhaps that e-mail is not spam after all.

doi:10.1038/nrendo.2010.48

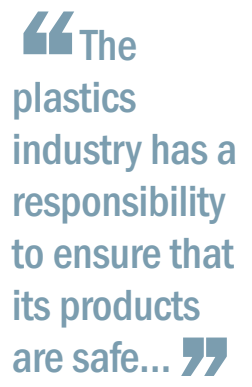

Vicky Heath is the Editor of Nature Reviews Endocrinology.

Competing interests The author declares no competing interests. 